\title{
Sinusoidal pumping of groundwater near cylindrical inhomogeneities
}

\author{
Mark Bakker
}

Received: 4 October 2007 / Accepted: 20 August 2008 / Published online: 19 September 2008

(C) The Author(s) 2008. This article is published with open access at Springerlink.com

\begin{abstract}
A new analytic-element solution is presented for a well with a sinusoidal discharge pumping near cylindrical inhomogeneities. Both the transmissivity and the storage coefficient may be different inside each cylinder. The solution consists of separate series solutions inside and outside each cylinder; asymptotic expansions are presented for cases where the sinusoidal period is small and the radius and transmissivity of the cylinder are large. The complex coefficients in the series solutions are determined by requiring continuity of head and normal flow across the boundary of each cylinder resulting in a linear system of complex equations that may be solved with standard methods. The solution represents one of the few analytic solutions for two-dimensional transient groundwater flow in an aquifer with inhomogeneities. Computer implementation requires truncation of the series; machine accuracy may be reached when enough terms are used in the series solutions. The effect of cylindrical inhomogeneities on the amplitude and phase of the head are investigated. The complex behavior suggests it may be difficult to determine location and properties of cylindrical inhomogeneities from pump tests, which confirms reported difficulties of interpreting pump test results from heterogeneous aquifers.
\end{abstract}

Keywords Analytic elements · Cylindrical inhomogeneities · Periodic flow

\section{Introduction}

The topic of this paper is the simulation of groundwater flow to wells with sinusoidal discharges in a porous-media aquifer containing an arbitrary number of cylindrical inhomogeneities. Inside each cylinder, a different value of both the transmissivity and storage coefficient may be specified. The solution consists of separate series solutions for the inside and the outside of each cylinder and is obtained with the analytic-element method. The solution is accurate up to machine accuracy provided that enough terms are used in the series solutions.

The analytic-element method has been applied successfully to solve several groundwater-flow problems with cylindrical inhomogeneities; solutions for inhomogeneities of other shapes exist as well but are not reviewed here. Strack [1] presented an analytic-element solution for steady confined and unconfined flow through cylindrical inhomogeneities. Barnes and Janković [2] extended this solution by providing expressions for the coefficients in the solution in terms of Fourier integrals and developed an iterative solution procedure that allowed for the solution

M. Bakker $(\bowtie)$

Water Resources Section, Faculty of Civil Engineering and Geosciences, Delft University of Technology, Delft, The Netherlands e-mail: mark.bakker@tudelft.nl 
of aquifers with many $\left(>10^{5}\right)$ inhomogeneities. Furman et al. [3] applied the same solutions to model electrical potentials in the subsurface for use with electrical-resistance tomography. Warrick and Knight [4] presented a solution for unsaturated flow through a single circular inhomogeneity in an otherwise uniform flow field. Bakker and Nieber [5] extended this solution by including an arbitrary number of circular inhomogeneities and allowing the water-retention parameters to differ between the inside and outside of the circles. Bakker [6] presented an analyticelement solution for steady multi-aquifer flow through many cylindrical inhomogeneities. Butler and Liu [7] solved the problem of a well that turns on at $t=0$ near a single circular inhomogeneity; they obtained explicit equations for the coefficients of the Laplace-domain solution, and transformed back to the physical domain with the Stehfest algorithm. Furman and Neuman [8] and Kuhlman and Neuman [9] obtained analytic-element solutions for transient flow in aquifers with multiple cylindrical inclusions by application of the analytic-element method in the Laplace domain and using a numerical back-transformation.

Many of the analytic-element solutions mentioned above are exact solutions consisting of infinite series; coefficients in the series are obtained with Fourier integrals or by truncating the series and solving a system of linear equations. Only a few solutions may be written in closed form without a series of infinite terms, including a steady well pumping near a single cylindrical inhomogeneity (e.g., [10] or [11, Eq. 370.04]), and uniform flow through a single cylindrical inhomogeneity in either a single aquifer (e.g., [10] or [11, Eq.370.05]) or a multi-aquifer system [12]. Exact transient solutions for problems involving wells and inhomogeneities are limited. Sternberg [13], Streltsova [14, Sect. 5.10] and Trefry [15] give solutions for radial flow, while Streltsova [14, Chap. 5] also provides solutions for wells pumping near straight inhomogeneity boundaries. Chu and Grader [16] give an overview of existing solutions in the Laplace domain for wells near inhomogeneities.

In this paper, an analytic-element solution is presented for a periodic well in a field of many cylindrical inhomogeneities. Groundwater-flow solutions with periodic boundary conditions are useful for the modeling of aquifers under the influence of tides, wells with sinusoidal discharges, or boundary conditions that may be represented as a Fourier series through time. Aquifer parameters may be determined by measuring the damping and phase-shift of tides (e.g., $[17,18])$ or through sinusoidal pump tests [19,20]. Many solutions exist for one-dimensional periodic flow but they are not reviewed here. The number of solutions for two-dimensional periodic groundwater flow is much smaller. Sun [21] and Tang and Jiao [22] published solutions where the boundary condition along the straight coast is a damped Fourier series. Solutions for L-shaped aquifers were published in [23] and [24]. Jeng et al. [25] developed a solution for the case where the shape of the coastal boundary is a sinusoidal function of space and included the effect of capillarity. A general analytic-element approach using point sinks, line-sinks, and circular area sinks was developed in [26]. While all referenced two-dimensional solutions are for homogeneous aquifers, Trefry [15] presented one-dimensional solutions for sinusoidal flow in aquifers consisting of either linear or radial strips with different aquifer properties. The analytic-element solution presented in this paper may be viewed as an extension of the work of Trefry [15] to two-dimensions with cylindrical inhomogeneities. It is an accepted fact that "the properties of heterogeneous media vary spatially in a manner that can seldom be described with certainty" [27]. This paper will highlight this problem by showing the complex transient head variations due to sinusoidal pumping near cylindrical inhomogeneities.

\section{Problem description}

Consider an arbitrary number of homogeneous, non-overlapping, cylindrical inclusions embedded in an otherwise homogeneous, horizontal background aquifer. The inside of cylinder $j$ is referred to as domain $\mathcal{D}_{j}$ while the background aquifer is referred to as domain $\mathcal{D}_{0}$. The hydraulic conductivity and storage coefficient of cylinder $j$ are called $k_{j}$ and $S_{j}$, respectively; the index $j=0$ is used for the properties of the background aquifer. For confined flow, the aquifer diffusivity is defined as

$D_{j}=k_{j} H_{j} / S_{j}=T_{j} / S_{j}$ in $\mathcal{D}_{j}$, 
where $H_{j}$ is the aquifer thickness and $T_{j}=k_{j} H_{j}$ is the transmissivity in domain $j$. For unconfined flow, the aquifer diffusivity is approximated as

$D_{j}=k_{j} \bar{h}_{j} / S_{j}=T_{j} / S_{j}$ in $\mathcal{D}_{j}$,

where $\bar{h}_{j}$ is an approximation of the average saturated thickness in domain $j$ and $T_{j}=k_{j} \bar{h}_{j}$ is the average transmissivity. Note that flow in each domain is treated as either confined or unconfined; cylinders with confined flow may be embedded in a background of unconfined flow or vice versa.

The Dupuit approximation is adopted so that the hydraulic head $h$ is a function of the two horizontal coordinates $x$ and $y$ only. The vertically integrated discharge vector $\vec{Q}$ may be written as

$\vec{Q}=-\nabla \Phi$,

where $\Phi$ is the discharge potential defined as

$\Phi=T_{j} h$ in $\mathcal{D}_{j}$,

where $h$ is the hydraulic head. For transient flow, the discharge potential fulfills the standard heat equation (e.g., [28, Sect. 16])

$\nabla^{2} \Phi=\frac{1}{D_{j}} \frac{\partial \Phi}{\partial t}$ in $\mathcal{D}_{j}$

where $\nabla^{2}$ is the two-dimensional Laplacian and $t$ is time. For unconfined flow, Eq. 5 is known as the linearized Boussinesq equation. Although it is common practice to use solutions to (5) for the approximation of unconfined flow, the author is not aware of any studies that assess the accuracy of these approximate solutions for transient flow through piecewise homogeneous aquifers.

In this paper, all forcing functions vary sinusoidally through time which means that all heads and flows vary sinusoidally and no initial conditions need to be specified. Flow in the aquifer is caused by one or more pumping wells with a sinusoidal discharge. In the absence of sinusoidal wells, flow is at steady state and thus the solution presented here is a deviation from the steady-state solution. Other features such as line-sinks with a sinusoidal discharge or sinusoidal areal recharge are not included here but are described in [26]. It is noted that, for unconfined flow, the potential may alternatively be defined in such a way that the dependence of the saturated thickness on the hydraulic head is taken into account (e.g., [28, Sect. 7]), which results in the correct steady head inside the well, even while taking into account the seepage face (e.g., [29, Eq. 9.13]). Definition (4) is a common and accurate approximation when the head variation is small relative to the saturated thickness, and it facilitates evaluation of the individual contribution of each periodic stress.

Across the boundary of each cylinder there are two connecting conditions: the head is continuous and the component of flow normal to the boundary is continuous. For cylinder $j$ with radius $R_{j}$ this may be written as

$T_{0} \Phi^{+}\left(r=R_{j}\right)=T_{j} \Phi^{-}\left(r=R_{j}\right), \quad Q_{r}^{+}\left(r=R_{j}\right)=Q_{r}^{-}\left(r=R_{j}\right)$,

where $r$ is the radial coordinate originating at the center of cylinder $j, Q_{r}$ is the component of the discharge vector in this direction, and the superscripts + and - stand for evaluation just inside and just outside cylinder $j$, respectively.

\section{Solution approach}

A solution to the stated problem is obtained with the analytic-element method; a recent overview of the analyticelement method is given in [30]. An analytic-element solution consists of the superposition of analytic solutions to the differential equation. Each analytic solution has one or more degrees of freedom that are determined from desired boundary conditions. A framework for analytic-element solutions for periodic flow is presented in [26].

A solution to the stated problem is derived for a single period $p$. The solution for multiple periods may be obtained through superposition. Periodic flow solutions are commonly derived in complex form (e.g., $[31,11,19,26])$ where the discharge potential $\Phi$ is written as the real part of a complex function $\Omega$

$\Phi=\mathfrak{R e} \Omega$. 
The complex function $\Omega$ is written in separated form

$\Omega=\omega \mathrm{e}^{\mathrm{i} \theta t}$,

where $\omega$ is a complex function of the spatial coordinates only, and

$\theta=2 \pi / p$,

where $p$ is the time period of oscillation. Substitution of $\Omega(8)$ for $\Phi$ in (5) and cancelling the exponential terms results in the modified Helmholtz equation for $\omega$

$\nabla^{2} \omega=\omega i \theta / D_{j}$ in $\mathcal{D}_{j}$.

The solution for an aquifer with $N_{w}$ wells and $M_{c}$ cylindrical inclusions is obtained through superposition

$\omega=\sum_{n=1}^{N_{w}} \omega_{w, n}+\sum_{m=1}^{M_{c}} \omega_{c, m}$

where $\omega_{w, n}$ and $\omega_{c, m}$ are the solutions for well $n$ and cylinder $m$, respectively. The real and imaginary parts of the complex function $\omega$ are written as $\phi$ and $\psi$, respectively

$\omega=\phi+\mathrm{i} \psi$.

When $\omega$ is known, the potential at any point and any time may be computed with (7), (8) and (12) as

$\Phi=\phi \cos (\theta t)-\psi \sin (\theta t)$.

The head $h$ in $\mathcal{D}_{j}$ may be obtained through division of $\Phi$ by transmissivity $T_{j}$. It is often mainly of interest to compute the amplitude, $\operatorname{amp}(h)$, and phase, $\arg (h)$, of the head, which may be written as

$\operatorname{amp}(h)=\sqrt{\phi^{2}+\psi^{2}} / T_{j}$ in $\mathcal{D}_{j}$

and

$\arg (h)=\arctan (\psi / \phi)$.

The solution for a single well or cylindrical inhomogeneity may both be written in local, cylindrical coordinates $r$ and $\alpha$, with the origin either at the center of the well or the center of the cylindrical inhomogeneity. A general solution to (10) for $\omega$ in cylindrical coordinates $r$ and $\alpha$ is given by, e.g., [32, p. 16] as

$\omega=\sum_{n=0}^{\infty}\left[\mathcal{A}_{n} \mathrm{I}_{n}(r \sqrt{\mathrm{i} \theta / D})+\mathcal{B}_{n} \mathrm{~K}_{n}(r \sqrt{\mathrm{i} \theta / D})\right]\left[\mathcal{P}_{n} \cos (n \alpha)+\mathcal{Q}_{n} \sin (n \alpha)\right]$,

where $\mathcal{A}_{n}, \mathcal{B}_{n}, \mathcal{P}_{n}, \mathcal{Q}_{n}$ are complex constants, and $\mathrm{I}_{n}$ and $\mathrm{K}_{n}$ are modified Bessel functions of order $n$ and of the first and second kind, respectively.

The discharge vector $\vec{Q}$ is obtained from (3) and (13) as

$\vec{Q}=-\nabla \phi \cos (\theta t)+\nabla \psi \sin (\theta t)$.

The complex vector $\vec{w}$ is introduced as

$\vec{w}=-\nabla \omega$.

The components of $\vec{w}$ in the local $r, \alpha$ coordinate system are called $u$ and $v$

$\vec{w}=u \vec{e}_{r}+v \vec{e}_{\alpha}$,

where $\vec{e}_{r}$ and $\vec{e}_{\alpha}$ are unit vectors in the $r$ and $\alpha$ directions, respectively, and, using (12),

$u=-\frac{\partial \omega}{\partial r}=-\frac{\partial \phi}{\partial r}-\mathrm{i} \frac{\partial \psi}{\partial r}, \quad v=-\frac{1}{r} \frac{\partial \omega}{\partial \alpha}=-\frac{1}{r}\left(\frac{\partial \phi}{\partial \alpha}+\mathrm{i} \frac{\partial \psi}{\partial \alpha}\right)$. 
The radial components of the discharge vector may now be written as

$Q_{r}=-\frac{\partial \Phi}{\partial r}=\mathfrak{R e} u \cos (\theta t)-\mathfrak{I m} u \sin (\theta t)$.

The connecting conditions across the boundary of cylinder $j(6)$ need to be fulfilled for all times. The potential $\Phi(13)$ is a function of both the real part $(\phi)$ and the imaginary part $(\psi)$ of $\omega$. Similarly, the radial component of flow (21) is a function of both the real and imaginary parts of $u$. Hence, conditions (6) will be fulfilled for all time when the following conditions for the complex functions $\omega$ and $u$ are met

$T_{0} \omega^{+}\left(r=R_{j}\right)=T_{j} \omega_{j}^{-}\left(r=R_{j}\right)$

$u^{+}\left(r=R_{j}\right)=u^{-}\left(r=R_{j}\right)$.

Note that the tangent component $v$ is discontinuous across an inhomogeneity boundary.

\section{Solution for a well}

Consider a fully penetrating well with a sinusoidal discharge with amplitude $Q_{0}$

$Q=Q_{0} \cos (\theta t)$.

The solution for a well with an infinitesimal radius and no skin effect is given in, e.g., [14]; application of the presented solutions to a well with a finite radius and skin effect is outlined in Sect. 8. Well $n$ is located in domain $\mathcal{D}_{j}$. The solution is set to zero in the domains other than $\mathcal{D}_{j}$ and is written in terms of $\omega$

$\omega_{w, n}=-\frac{Q_{0}}{2 \pi} \mathrm{K}_{0}\left(\rho_{j} \sqrt{\mathrm{i}}\right)$ in $\mathcal{D}_{j}$,

$\omega_{w, n}=0$ in $\mathcal{D}_{m}, \quad m \neq j$,

where $\rho_{j}$ is a scaled radial coordinate introduced for convenience

$\rho_{j}=r \sqrt{\theta / D_{j}}$.

The radial component $u$ of $\vec{w}$ for well $n$ is

$u_{w, n}=-\frac{Q_{0}}{2 \pi} \sqrt{\mathrm{i} \theta / D_{j}} \mathrm{~K}_{1}\left(\rho_{j} \sqrt{\mathrm{i}}\right)$ in $\mathcal{D}_{j}$,

$u_{w, n}=0$ in $\mathcal{D}_{m}, m \neq j$,

while the tangential component $v_{w, n}$ is zero everywhere.

In a homogeneous aquifer, the amplitude decreases with distance from the pumping wells. This damping of the amplitude is a function of the characteristic length $\lambda_{j}$, which is defined in domain $j$ as $([26,33])$

$\lambda_{j}=\sqrt{D_{j} / \theta}=\sqrt{\frac{T_{j} p}{2 \pi S_{j}}}$.

Note that $\lambda_{j}$ is a function of both the aquifer parameters $T_{j}$ and $S_{j}$, and the period $p$. The ratio between the amplitude at a distance $r$ and the amplitude at a distance $r=0.1 \lambda_{j}$ is shown in Fig. 1 (solid line, left axis). Also shown in Fig. 1 is the phase shift as compared to the phase of the discharge at the well (dashed line, right axis). Note that relative to the amplitude at $r=0.1 \lambda_{j}$, the amplitude has reduced to $10 \%$ at $r=1.782 \lambda_{j}$, to $1 \%$ at $r=4.432 \lambda_{j}$, and to $0.1 \%$ at $r=7.342 \lambda_{j}$.

\section{Solution for a cylindrical inhomogeneity}

An equation for cylinder $m$ is obtained from the general solution (16). Inside cylinder $m$, the diffusivity is $D_{j}$, and the potential is finite everywhere so that no functions $\mathrm{K}_{n}$ appear in the solution.

$\omega_{c, m}=a_{0} \beta_{0} \mathrm{I}_{0}\left(\rho_{j} \sqrt{\mathrm{i}}\right)+\sum_{n=1}^{\infty}\left[a_{n} \cos (n \alpha)+b_{n} \sin (n \alpha)\right] \beta_{n} \mathrm{I}_{n}\left(\rho_{j} \sqrt{\mathrm{i}}\right)$ in $\mathcal{D}_{j}$, 
Fig. 1 Damping of amplitude (solid line, left axis) and phase shift (dashed line, right axis) with distance from the well; amplitude normalized by value at $r=0.1 \lambda_{0}$

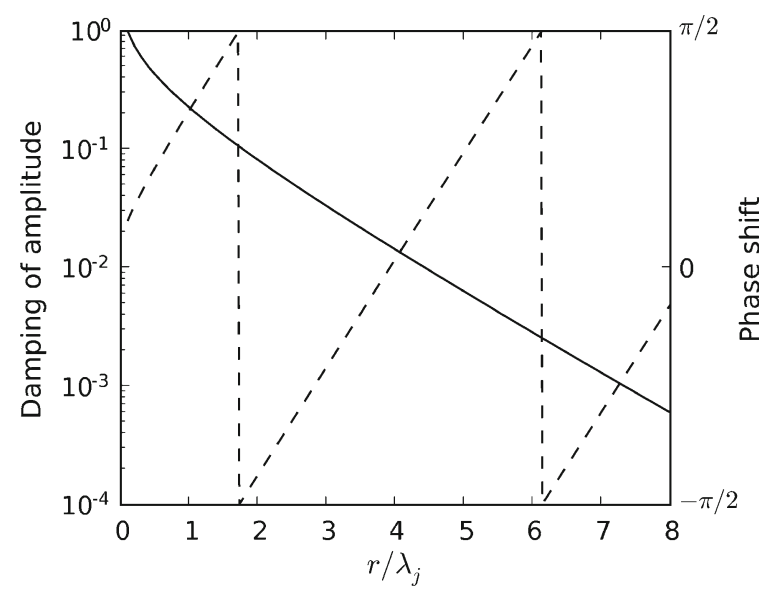

where $\beta_{n}=1 / \mathrm{I}_{n}\left(R \sqrt{\mathrm{i} \theta / D_{j}}\right)$ is a scaling parameter introduced for computational purposes, and $a_{n}$ and $b_{n}$ are complex coefficients to be determined from connecting conditions (22). In the background aquifer, the diffusivity is $D_{0}$, and the potential vanishes at infinity so that no functions $\mathrm{I}_{n}$ appear in the solution.

$\omega_{c, m}=A_{0} \gamma_{0} \mathrm{~K}_{0}\left(\rho_{0} \sqrt{\mathrm{i}}\right)+\sum_{n=1}^{\infty}\left[A_{n} \cos (n \alpha)+B_{n} \sin (n \alpha)\right] \gamma_{n} \mathrm{~K}_{n}\left(\rho_{0} \sqrt{\mathrm{i}}\right)$ in $\mathcal{D}_{0}$,

where $\gamma_{n}=1 / \mathrm{K}_{n}\left(R \sqrt{\mathrm{i} \theta / D_{0}}\right)$ is a scaling parameter introduced for computational purposes, and $A_{n}$ and $B_{n}$ are complex coefficients to be determined from the connecting conditions. Inside any of the other cylinders, the function $\omega_{j}$ is zero

$\omega_{c, m}=0$ in $\mathcal{D}_{n}, \quad n \neq 0, j$.

Note that the radius $R$ and the parameters $\beta_{n}, \gamma_{n}, a_{n}, b_{n}, A_{n}$, and $B_{n}$ are, in general, different for each cylinder, but double indices have not been introduced here to keep the notation simple.

The equations for the components of the vector $\vec{w}$ are obtained from (18) through differentiation as

$u_{c, m}=a_{0} \beta_{0} \mathrm{I}_{0}^{\prime}\left(\rho_{j} \sqrt{\mathrm{i}}\right)+\sum_{n=1}^{\infty}\left[a_{n} \cos (n \alpha)+b_{n} \sin (n \alpha)\right] \beta_{n} \mathrm{I}_{n}^{\prime}\left(\rho_{j} \sqrt{\mathrm{i}}\right)$ in $\mathcal{D}_{j}$,

$v_{c, m}=\sum_{n=1}^{\infty}\left[-a_{n} \sin (n \alpha)+b_{n} \cos (n \alpha)\right] \frac{n}{r} \beta_{n} \mathrm{I}_{n}\left(\rho_{j} \sqrt{\mathrm{i}}\right)$ in $\mathcal{D}_{j}$,

$u_{c, m}=A_{0} \gamma_{0} \mathrm{~K}_{0}^{\prime}\left(\rho_{0} \sqrt{\mathrm{i}}\right)+\sum_{n=1}^{\infty}\left[A_{n} \cos (n \alpha)+B_{n} \sin (n \alpha)\right] \gamma_{n} \mathrm{~K}_{n}^{\prime}\left(\rho_{0} \sqrt{\mathrm{i}}\right)$ in $\mathcal{D}_{0}$,

$v_{c, m}=\sum_{n=1}^{\infty}\left[-A_{n} \sin (n \alpha)+B_{n} \cos (n \alpha)\right] \frac{n}{r} \gamma_{n} \mathrm{~K}_{n}\left(\rho_{0} \sqrt{\mathrm{i}}\right)$ in $\mathcal{D}_{0}$,

$u_{c, m}=v_{c, m}=0$ in $\mathcal{D}_{n}, \quad n \neq 0, j$,

where the prime stands for differentiation with respect to $r$ (e.g., [34, Eq.9.6.26])

$\mathrm{I}_{n}^{\prime}\left(\rho_{j} \sqrt{\mathrm{i}}\right)=\frac{\mathrm{I}_{n-1}\left(\rho_{j} \sqrt{\mathrm{i}}\right)+\mathrm{I}_{n+1}\left(\rho_{j} \sqrt{\mathrm{i}}\right)}{2} \sqrt{\mathrm{i} \theta / D_{j}}$, 
$\mathrm{K}_{n}^{\prime}\left(\rho_{0} \sqrt{\mathrm{i}}\right)=-\frac{\mathrm{K}_{n-1}\left(\rho_{0} \sqrt{\mathrm{i}}\right)+\mathrm{K}_{n+1}\left(\rho_{0} \sqrt{\mathrm{i}}\right)}{2} \sqrt{\mathrm{i} \theta / D_{0}}$.

The complex coefficients $A_{n}, B_{n}, a_{n}$, and $b_{n}$ are obtained through application of the boundary conditions (22). In practice the series are truncated after $N_{t}$ terms so that there are $N_{t}+1$ unknowns $A_{n}$ and $a_{n}$ and $N_{t}$ unknowns $B_{n}$ and $b_{n}$. Boundary conditions (22) are applied at $2 N_{t}+1$ equally spaced collocation points along the boundary of the cylinder, resulting is a system of $4 N_{t}+2$ complex linear equations. The system of equations is solved using a standard LDU decomposition routine (the linalg. solve routine in the Python package numpy [35]). The effect of the number of terms $N_{t}$ on the accuracy of the solution is evaluated in the next section.

It is noted that the expression for a cylindrical inhomogeneity in $\mathcal{D}_{0}(29)$ may also be used to model cylindrical features with other boundary conditions, for example an impermeable object (by setting $u=0$ at all control points), or a circular lake with a constant but fluctuating level (by setting $v=0$ at all control points) or a circular lake with a constant level (by setting $\omega=0$ at all points) or a specified amplitude (by specifying the desired $\omega$ ). The solution for a well with infinitesimal radius may be combined with the solution for a cylindrical inhomogeneity to simulate a finite radius well with wellbore storage. This may be achieved by centering the cylinder at the well, setting the radius of the cylinder equal to the radius of the well, setting the storativity to one, and setting the transmissivity to a large value (for example 6 orders of magnitude larger than outside) so that the head inside the well is flat. Skin effect (resistance to inflow or outflow) may be added by replacing the connecting conditions along the boundary of the cylinder (6) with Cauchy (third-type) boundary conditions.

Use of common routines for the computation of modified Bessel functions are not suited for larger arguments. For example, $\mathrm{K}_{0}(\rho \sqrt{\mathrm{i}})$ returns zero for values of $\rho$ larger than 985 . Such arguments may occur for circles with large radii, short periods of the fluctuation, large transmissivities, or a combination thereof. Since only ratios of modified Bessel functions occur in the solution for a cylindrical inhomogeneity, asymptotic expansions may be used for cases where the common routines don't work, as explained in the Appendix.

\section{Well pumping near a single cylinder}

The effect of a single cylinder on the amplitude of a pumping well is investigated. The transmissivity in heterogeneous aquifers may vary over several orders of magnitude. A reduction of the storage coefficient by several orders indicates a change from unconfined to confined conditions. Six cases are considered. For all cases, the well and the center of the cylinder lie on the same horizontal line. The edge of the cylinder is a distance $0.5 \lambda_{0}$ from the well, and the radius of the cylinder is $\lambda_{0}$. The aquifer properties on the inside of the cylinder are listed in Table 1 for all six cases. Table 1 has four columns listing the values of $T_{1}, S_{1}, \lambda_{1}$, and $D_{1}$; only two of these values may be chosen independently. Contour plots of the amplitude are shown in Fig. 2 and are normalized through division by the amplitude at a distance of $0.1 \lambda_{0}$ from the well in the absence of the cylinder. In Fig. 3, the variation of the amplitude is shown as a function of distance from the well along the horizontal line that goes through the center of the well and the cylinder. At each point, the amplitude is divided by the amplitude at the same point corresponding to the case without the cylinder. Hence, a value of 2 means that the amplitude is twice as large for the case with the inhomogeneity as for the case without the inhomogeneity. In Fig. 4, the phase is shown along the same horizontal line; for comparison purposes, the phase for the homogeneous case is shown in the top graph of Fig. 4. For each case, 41 terms are used in the series solution.

For cases 1 and 2 the transmissivity inside the cylinder is changed while the storage coefficient is kept the same as the background. For case 1, the transmissivity of the cylinder is 100 times larger than the background. This results in a strong damping between the well and the edge of the cylinder. Inside the cylinder, the amplitude is fairly constant, and thus increases with respect to the homogeneous case, so that at the back of the cylinder the amplitude is more than 2.5 times larger than for the homogeneous case. Inside the cylinder, the phase is almost constant. For case 2, the transmissivity of the cylinder is 100 times lower than the background, and the opposite happens from case 1 . The amplitude dampens less between the well and the edge of the cylinder than in the homogeneous case, but 
Table 1 Aquifer properties used in Figs. 2-4

\begin{tabular}{lllll}
\hline Case & $T_{1}$ & $S_{1}$ & $\lambda_{1}$ & $D_{1}$ \\
\hline 1 & $100 T_{0}$ & $S_{0}$ & $10 \lambda_{0}$ & $100 D_{0}$ \\
2 & $0.01 T_{0}$ & $S_{0}$ & $0.1 \lambda_{0}$ & $0.01 D_{0}$ \\
3 & $T_{0}$ & $100 S_{0}$ & $0.1 \lambda_{0}$ & $0.01 D_{0}$ \\
4 & $T_{0}$ & $0.01 S_{0}$ & $10 \lambda_{0}$ & $100 D_{0}$ \\
5 & $100 T_{0}$ & $100 S_{0}$ & $\lambda_{0}$ & $D_{0}$ \\
6 & $0.01 T_{0}$ & $0.01 S_{0}$ & $\lambda_{0}$ & $D_{0}$ \\
\hline
\end{tabular}
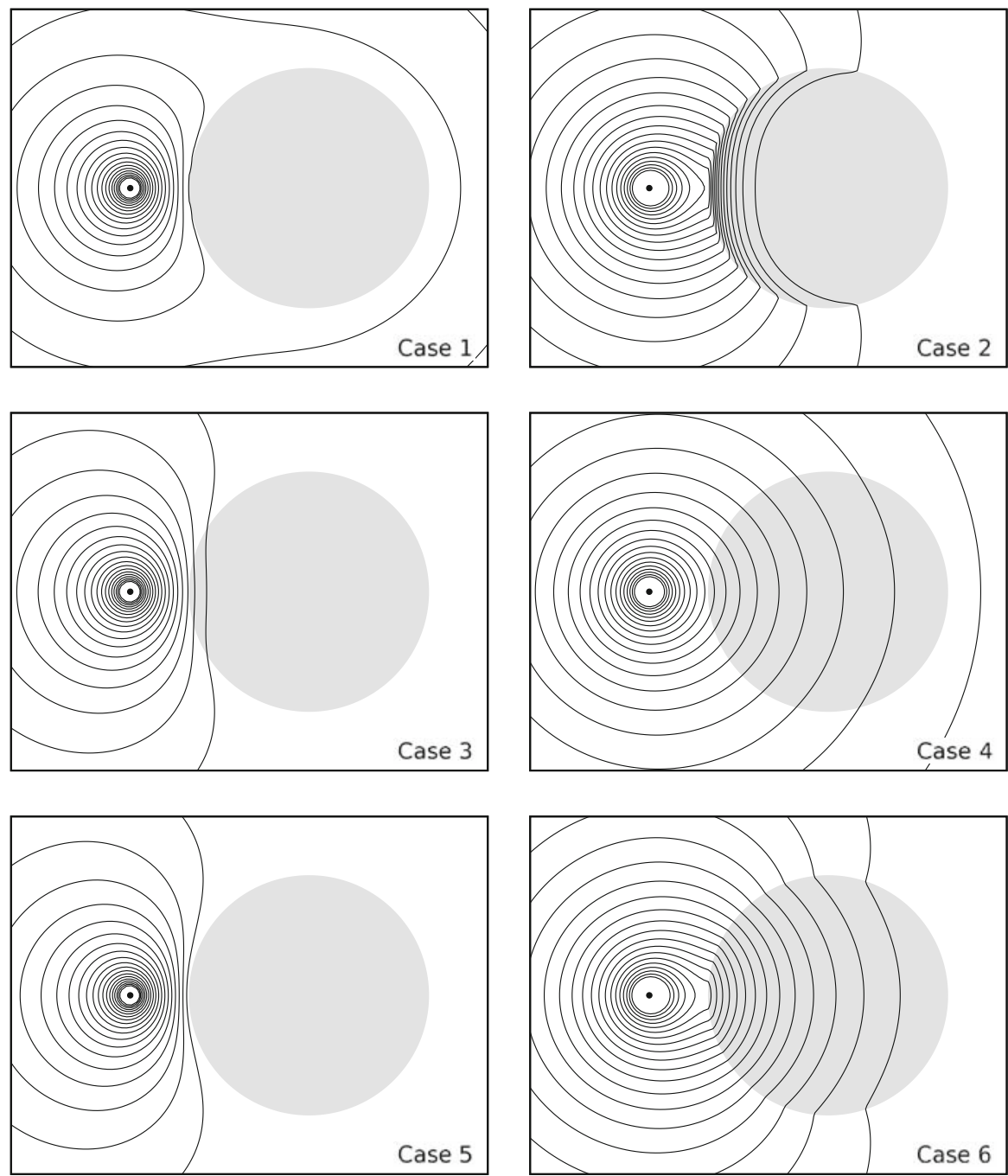

Fig. 2 Contour plots of amplitude of head for six cases of a well pumping near a cylindrical inhomogeneity; amplitude normalized by value at $0.1 \lambda_{0}$ from well for homogeneous case. The value along contour farthest away from the well is 0.05 ; the contour interval is 0.05

dampens very quickly inside the cylinder; near the back of the cylinder there is a small recovery of the amplitude. The phase also changes quickly inside the cylinder, going through approximately four cycles.

For cases 3 and 4 the storage coefficient inside the cylinder is changed while the transmissivity is kept the same as the background. For case 3 the storage coefficient is increased 100 times as compared to the background. This has a very strong damping effect on the amplitude, resulting in almost no amplitude beyond a sliver of the cylinder 

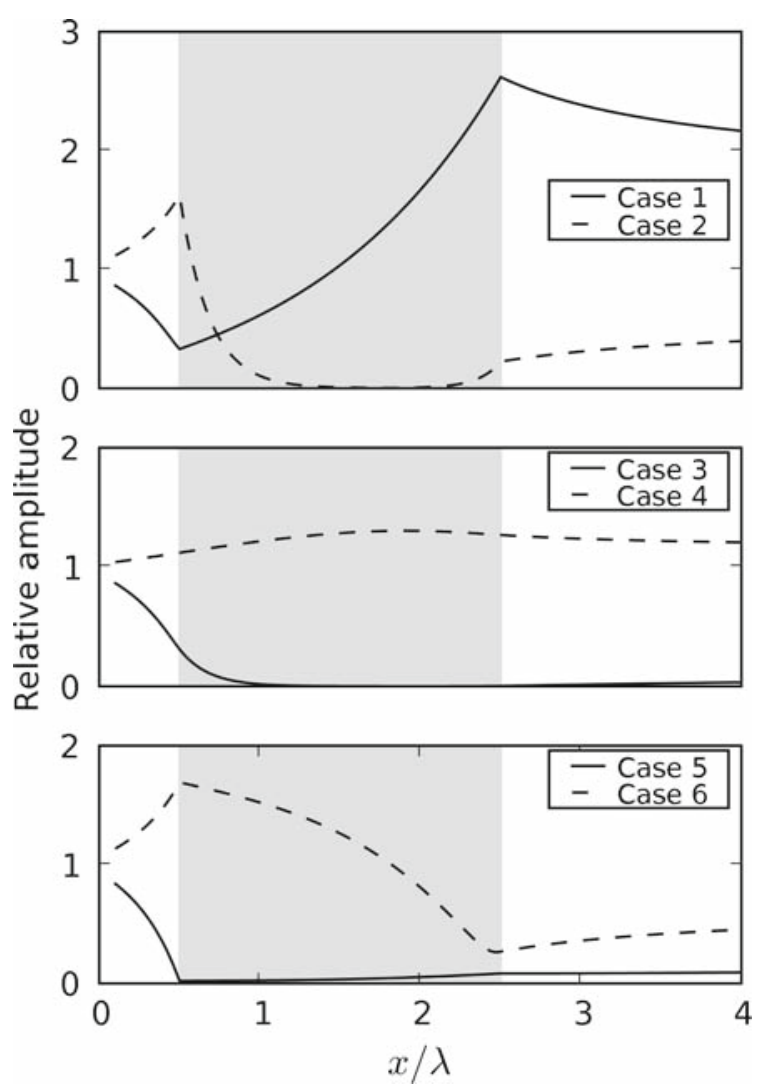

Fig. 3 Amplitude of head along $x$-axis normalized by homogeneous case versus distance from the well along line through center of cylinder
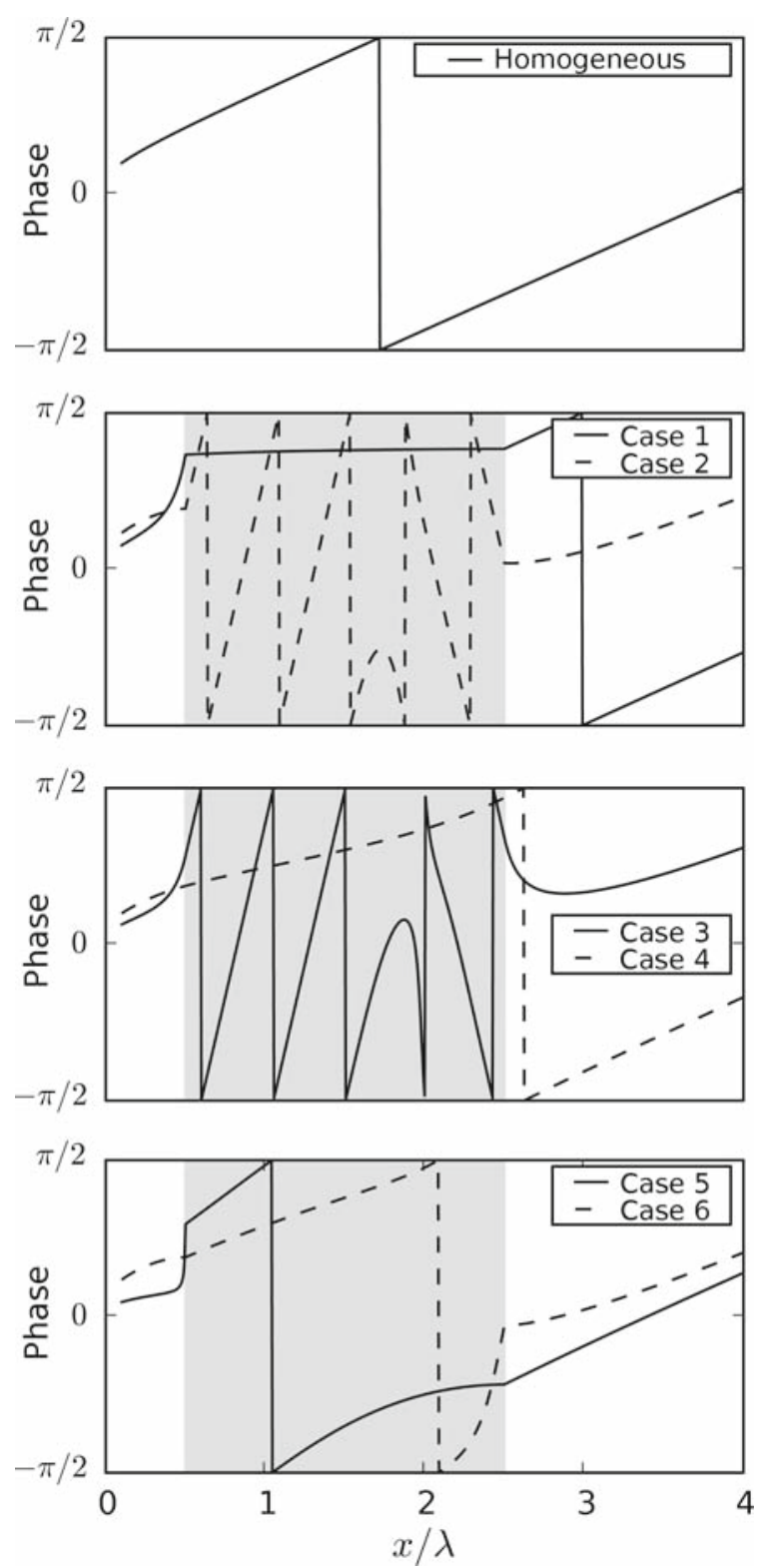

Fig. 4 Phase of head along $x$-axis versus distance from the well along line through center of cylinder

closest to the well. A hundred-fold decrease in the storage coefficient from the background (case 4) has the opposite effect, but is much less pronounced. The amplitude is slightly larger than for the homogeneous case and the phase changes over a slightly larger distance, but the deviations from the homogeneous case are minor. Note that for both cases 3 and 4, the tangential derivative of the contour lines of the amplitude are continuous across the boundary of the cylinder, as the transmissivity inside the cylinder is the same as the background.

For cases 5 and 6 , the aquifer diffusivity (and thus the characteristic length $\lambda$ ) inside the cylinder is the same as the background. For case 5, both the transmissivity and the storage coefficient are increased by a factor 100 from the background. This results in the strongest damping between the well and the edge of the cylinder. Inside the 
Table 2 Accuracy of case 1

\begin{tabular}{lllll}
\hline$N_{t}$ & $\overline{\left|\varepsilon_{1}\right|}$ & $\overline{\left|\varepsilon_{2}\right|}$ & $\operatorname{Max}\left|\varepsilon_{1}\right|$ & $\operatorname{Max}\left|\varepsilon_{2}\right|$ \\
\hline 10 & $1.8 \times 10^{-4}$ & $1.3 \times 10^{-3}$ & $1.4 \times 10^{-3}$ & $1.1 \times 10^{-2}$ \\
20 & $1.7 \times 10^{-6}$ & $2.2 \times 10^{-5}$ & $1.3 \times 10^{-5}$ & $1.9 \times 10^{-4}$ \\
30 & $1.9 \times 10^{-8}$ & $3.8 \times 10^{-7}$ & $1.6 \times 10^{-7}$ & $3.3 \times 10^{-6}$ \\
40 & $2.5 \times 10^{-10}$ & $6.5 \times 10^{-9}$ & $2.1 \times 10^{-9}$ & $5.8 \times 10^{-8}$ \\
50 & $3.3 \times 10^{-12}$ & $1.0 \times 10^{-10}$ & $3.0 \times 10^{-11}$ & $1.0 \times 10^{-9}$ \\
60 & $5.1 \times 10^{-14}$ & $2.0 \times 10^{-12}$ & $4.4 \times 10^{-13}$ & $1.7 \times 10^{-11}$ \\
\hline
\end{tabular}

cylinder there is a minor recovery; the phase change is not very different from the homogeneous case. For case 6 , both the transmissivity and the storage coefficient are decreased by a factor 100 from the background. This results in a decreased damping between the well and the edge of the cylinder (comparable to case 2). Inside the cylinder, there is more damping, but it is still not as significant as for case 2. The phase change is spread out over a somewhat larger distance than the homogeneous case, but the difference is small.

A finite number of $N_{t}$ terms is used in the series solution. The accuracy of the solution as a function of $N_{t}$ is investigated by considering case 1 . The error in the connecting conditions (22) is computed at 1,000 equally spaced points along the boundary of the cylinder for different values of $N_{t}$. The mean absolute error and the maximum absolute error are reported in Table 2; $\varepsilon_{1}$ refers to the error in $\omega$ and $\varepsilon_{2}$ refers to the error in $u$. It may be seen from Table 2 that 10 additional terms in the series solution results in approximately an increase in accuracy of two orders of magnitude until machine accuracy is reached.

\section{Multiple cylinders}

A case with multiple cylinders is solved to demonstrate the capabilities of the solution. For cases with many cylindrical inhomogeneities, it may not be possible to build one large matrix to solve for the complex coefficients of all cylinders simultaneously because of the computer memory required to store and solve the system of equations. For such cases, an iterative solution approach was developed in [2], where it was proposed to solve for the coefficients of one cylinder at a time while keeping the coefficients of the other cylinders fixed. A solution is obtained by going through all cylinders multiple times until the values of the coefficients stop changing. A similar approach is applied to solve for the problem presented here.

Consider a field with 36 cylindrical inhomogeneities; the order of each cylinder is 40 . The radius of each cylinder is equal to $\lambda_{0}$, while the distance between each cylinder is $0.2 \lambda_{0}$. The transmissivity inside each cylinder is 100 times less than the background transmissivity and the storage coefficient is the same as the background aquifer. The well is located at the center of Fig. 5. The contour lines of the amplitude in Fig. 5 are again divided by the value at a distance of $0.1 \lambda_{0}$ from the well for the homogeneous case.

\section{Conclusions and discussion}

An analytic-element solution has been presented for periodic flow in confined and unconfined aquifers with multiple cylindrical inhomogeneities in both the transmissivity and the storage coefficient; the flow may be confined inside a cylinder while it is unconfined outside, or vice versa. The flow in the aquifer is caused by a well with a sinusoidal discharge but other features such as line-sinks may be added following the approach described in [26]. Separate series solutions were used for the inside and the outside of each cylinder. A system of linear equations needs to be solved to obtain the complex coefficients in the series. Asymptotic expressions have been presented to evaluate the ratio of modified Bessel functions in the series solution for cylinders with a large dimensionless radius. The connecting condition across the boundary of the inhomogeneity is met up to machine accuracy provided that enough 
Fig. 5 Contour lines of normalized amplitude for a well in a field of 36 inhomogeneities with $T_{j}=0.01 T_{0}$. The value along contour farthest from well is 0.05 ; the contour interval is 0.05

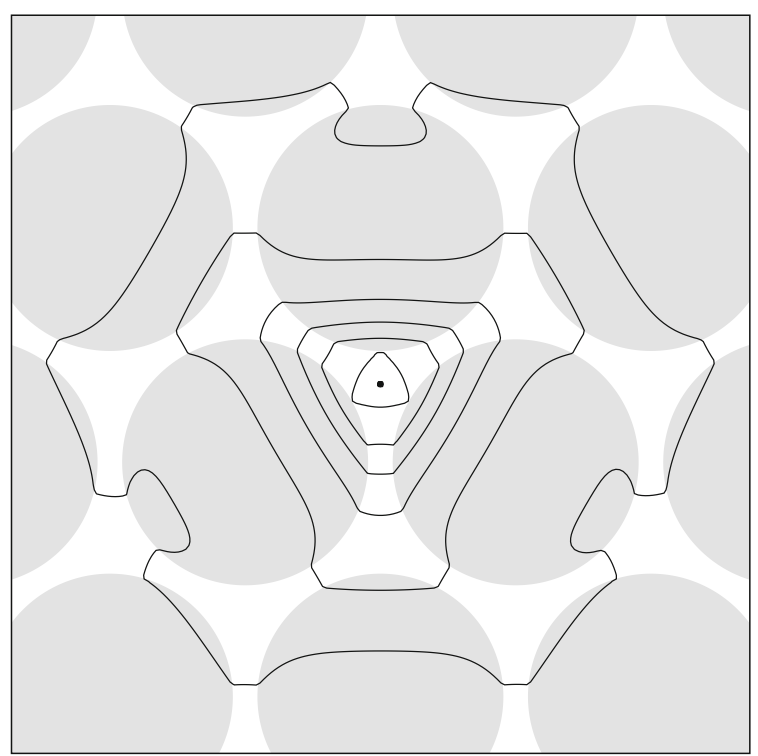

terms are used in the series. The amplitude and phase of the head may vary in a complicated fashion even for a well near a single cylindrical inhomogeneity, which may make it a challenge to analyze sinusoidal pumping tests with only a few observations and an imprecise idea of the location of the inhomogeneities. Arbitrary discharge functions may be represented with Fourier series; block-shaped discharge functions may be approximated with Fourier series in combination with Cesaro summation [36].

It was outlined how the presented solution for a well and a cylindrical inhomogeneity may be combined to simulate flow to a finite-radius well with wellbore storage and skin effect. The solutions for the outside of a cylinder may also be used to model impermeable cylinders or cylinders with other boundary conditions such as a specified head fluctuation. The solution for periodic flow through cylindrical inhomogeneities in semi-confined aquifers or multi-aquifer systems consists of a similar series of geometric functions multiplied by modified Bessel functions as (28) and (29). The solution approach may also be applied to two-dimensional periodic unsaturated flow and three-dimensional periodic flow.

Acknowledgements The author thanks Kees Maas for his suggestion to use the reciprocity principle [11, p. 823] to check the computer implementation of the solution.

Open Access This article is distributed under the terms of the Creative Commons Attribution Noncommercial License which permits any noncommercial use, distribution, and reproduction in any medium, provided the original author(s) and source are credited.

\section{Appendix: Evaluation of functions for large radii}

When the argument of the modified Bessel functions becomes large, they cannot be evaluated with common implementations in computer libraries, but asymptotic expansions need to be used. This occurs when the dimensionless radius $R=R_{j} \sqrt{\mathrm{i} \theta / D_{j}}$ is large. Asymptotic expansions of the modified Bessel functions may be written using Hankel symbols defined as (e.g., [37, p.238])

$(v, n)=\frac{\left(4 v^{2}-1\right)\left(4 v^{2}-3^{2}\right) \ldots\left[4 v^{2}-(2 n-1)^{2}\right]}{n ! 4^{n}}$

with $(\nu, 0)$ defined as $(v, 0)=1$ For the modified Bessel functions, the asymptotic expansions are [34, Eqs. 9.7.1 and 9.7.2] 
$\mathrm{I}_{n}(z)=\frac{\mathrm{e}^{z}}{\sqrt{2 \pi z}} \sum_{k=0}^{\infty}(-)^{k} \frac{(n, k)}{(2 z)^{k}}$,

$\mathrm{K}_{n}(z)=\sqrt{\frac{\pi}{2 z}} \mathrm{e}^{-z} \sum_{k=0}^{\infty} \frac{(n, k)}{(2 z)^{k}}$.

The derivatives of the modified Bessel functions are compiled from [34, Eqs. 9.7.3 and 9.7.4 with Eqs. 9.2.15 and 9.2.16]

$$
\begin{aligned}
& \mathrm{I}_{n}^{\prime}(z)=\frac{\mathrm{e}^{z}}{\sqrt{2 \pi z}}\left[\sum_{k=0}^{\infty} \mu_{k} \frac{(n, 2 k)}{(2 z)^{2 k}}-\sum_{k=0}^{\infty} v_{k} \frac{(n, 2 k+1)}{(2 z)^{2 k+1}}\right], \\
& \mathrm{K}_{n}^{\prime}(z)=-\sqrt{\frac{\pi}{2 z}} \mathrm{e}^{-z}\left[\sum_{k=0}^{\infty} \mu_{k} \frac{(n, 2 k)}{(2 z)^{2 k}}+\sum_{k=0}^{\infty} v_{k} \frac{(n, 2 k+1)}{(2 z)^{2 k+1}}\right],
\end{aligned}
$$

where

$\mu_{k}=\frac{4 n^{2}+16 k^{2}-1}{4 n^{2}-(4 k-1)^{2}}, \quad v_{k}=\frac{4 n^{2}+4(2 k+1)^{2}-1}{4 n^{2}-(4 k+1)^{2}}$.

The Bessel functions and their derivatives always appear in scaled form in (28) through (35). Note that shorthand is used in these equations, such as $\beta_{n} \mathrm{I}_{n}(\rho \sqrt{\mathrm{i}})$. A finite number of $M$ terms is used in the asymptotic expansions. Four types of ratios appear in the formulas, and may be approximated as

$$
\begin{aligned}
& \frac{\mathrm{I}_{n}(\rho \sqrt{\mathrm{i}})}{\mathrm{I}_{n}(R \sqrt{\mathrm{i}})} \approx \frac{\sum_{k=0}^{M}(-)^{k} \frac{(n, k)}{(2 \rho \sqrt{\mathrm{i}})^{k}}}{\sum_{k=0}^{M}(-)^{k} \frac{(n, k)}{(2 R \sqrt{\mathrm{i}})^{k}}} \sqrt{\frac{R}{\rho}} \mathrm{e}^{(\rho-R) \sqrt{\mathrm{i}}}, \quad R \gg 1, \\
& \frac{\mathrm{K}_{n}(\rho \sqrt{\mathrm{i}})}{\mathrm{K}_{n}(R \sqrt{\mathrm{i}})} \approx \frac{\sum_{k=0}^{M} \frac{(n, k)}{(2 z)^{k}}}{\sum_{k=0}^{M} \frac{(n, k)}{(2 R \sqrt{\mathrm{i}})^{k}} \sqrt{\frac{R}{\rho}} \mathrm{e}^{(R-\rho) \sqrt{\mathrm{i}}}, \quad R \gg 1,} \\
& \frac{\mathrm{I}_{n}^{\prime}(\rho \sqrt{\mathrm{i}})}{\mathrm{I}_{n}(R \sqrt{\mathrm{i}})} \approx \frac{\sum_{k=0}^{M} \mu_{k} \frac{(n, 2 k)}{(2 z)^{2 k}}-\sum_{k=0}^{M} v_{k} \frac{(n, 2 k+1)}{(2 z)^{2 k+1}}}{\sum_{k=0}^{M}(-)^{k} \frac{(n, k)}{(2 R \sqrt{\mathrm{i}})^{k}}} \sqrt{\frac{R}{\rho}} \mathrm{e}^{(\rho-R) \sqrt{\mathrm{i}}, \quad R \gg 1,} \\
& \frac{\mathrm{K}_{n}^{\prime}(\rho \sqrt{\mathrm{i}})}{\mathrm{K}_{n}(R \sqrt{\mathrm{i}})} \approx \frac{\sum_{k=0}^{M} \mu_{k} \frac{(n, 2 k)}{(2 z)^{2 k}}+\sum_{k=0}^{M} v_{k} \frac{(n, 2 k+1)}{(2 z)^{2 k+1}}}{\sum_{k=0}^{M} \frac{(n, k)}{(2 R \sqrt{\mathrm{i}})^{k}}} \sqrt{\frac{R}{\rho}} \mathrm{e}^{(R-\rho) \sqrt{\mathrm{i}}}, \quad R \gg 1 .
\end{aligned}
$$

As an indication, for (45) and $R=200,8$ significant digits are obtained for order $n=0$ using $M=1$ terms, while for order 20, 9 terms are needed and for order 40, 17 terms. It is noted that these expressions only need to be used within a certain distance from the boundary of the circle. For (45) and $R=200$, the ratio has reduced from 1 on the boundary to an absolute value less than $10^{-4}$ at $\rho=214$ for all orders up to 40 .

\section{References}

1. Strack ODL (1987) The analytic element method for regional groundwater modeling. In: Proceedings of the national water well association. Conference on solving groundwater problems with models. Denver, CO, 10-12 Feb 1987, 929-941.

2. Barnes R, Janković J (1999) Two-dimensional flow through large numbers of circular inhomogeneities. J Hydrol 226(3-4):204210

3. Furman A, Warrick AW, Ferré TyPA (2002) Electrical potential distributions in a heterogeneous subsurface in response to applied current: solution for circular inclusions. Vadose Zone J 1:273-280 
4. Warrick AW, Knight JH (2002) Two-dimensional unsaturated flow through a circular inclusion. Water Resour Res $38(7): 1113$. doi:10.1029/2001WR001041

5. Bakker M, Nieber JL (2004) Analytic element modeling of cylindrical drains and cylindrical inhomogeneities in steady twodimensional unsaturated flow. Vadose Zone J 3(3):1038-1049

6. Bakker M (2003) Steady groundwater flow through many cylindrical inhomogeneities in a multi-aquifer system. J Hydrol 277:268279

7. Butler JJ Jr, Liu W (1993) Pumping test in nonuniform aquifers: the radially asymmetric case. Water Resour Res 29(2):259-269

8. Furman A, Neuman SP (2003) Laplace-transform analytic element solution of transient flow in porous media. Adv Water Resour 26:1229-1237

9. Kuhlman KL, Neuman SP (2006) Recent advances in Laplace transform analytic element method (LT-AEM) theory and application to transient groundwater flow. In: Proceedings of computational methods in water resources, XVI, Copenhagen, Denmark, 19-22 June, 2006

10. Wheatcraft SW, Winterberg F (1985) Steady state flow passing through a cylinder of permeability different from the surrounding medium. Water Resour Res 21(12):1923-1929

11. Bruggeman GA (1999) Analytical solutions of geohydrological problems. Dev Water Sci 46, Elsevier

12. Bakker M (2002) Two exact solutions for a cylindrical inhomogeneity in a multi-aquifer system. Adv Water Resour 25(1):9-18

13. Sternberg YM (1969) Flow to wells in the presence of radial discontinuities. Ground Water 7:17-20

14. Streltsova TD (1988) Well testing in heterogeneous formations. Exxon monographs. Wiley, New York

15. Trefry MG (1999) Periodic forcing in composite aquifers. Adv Water Resour 22(6):645-656

16. Chu L, Grader AS (1999) Transient pressure and rate analysis for active and interference wells in composite systems. In Situ 23(4):297-332

17. Carr PA, Van der Kamp GS (1969) Determining aquifer characteristics by the tidal method. Water Resour Res 17(3):1023-1031

18. Trefry MG, Bekele E (2005) Structural characterization of an island aquifer via tidal methods. Water Resour Res 40(1):W01505. doi:10.1029/2003WR002003

19. Rasmussen TC, Haborak KG, Young MH (2003) Estimating aquifer hydraulic properties using sinusoidal pumping at the Savannah River site, South Carolina, USA. Hydrogeol J 11:466-482

20. Toll NJ, Bakker M, Rasmussen TC (2008) Sinusoidal testing of multi-layer aquifers. J Hydrol (Submitted)

21. Sun H (1997) A two-dimensional analytical solution of groundwater response to tidal loading in an estuary. Water Resour Res 33(6):1429-1435

22. Tang Z, Jiao JJ (2001) A two-dimensional analytical solution for groundwater flow in a leaky confined aquifer system near open tidal water. Hydrol Processes 15:573-585

23. Li H, Jiao JJ (2002) Tidal groundwater level fluctuations in L-shaped leaky coastal aquifer system. J Hydrol 268(1-4):234-243

24. Li H, Jiao JJ, Luk M, Cheung K (2002) Tide-induced groundwater level fluctuation in coastal aquifers bounded by L-shaped coastlines. Water Resour Res 38(3):1024. doi:10.1029/2001WR000556

25. Jeng D-S, Teo HT, Barry DA, Li L (2005) Two-dimensional approximation for tidal dynamics in coastal aquifers: capillarity correction. J Eng Mech 131(5):534-541

26. Bakker M (2004) Transient analytic elements for periodic Dupuit-Forchheimer flow. Adv Water Resour 27(1):3-12

27. Neuman SP, Blattstein A, Riva M, Tartakovsky DM, Guadagnini A, Ptak T (2007) Type curve interpretation of late-time pumping test data in randomly heterogeneous aquifers. Water Resour Res 43:W10421. doi:10.1029/2007WR005871

28. Strack ODL (1989) Groundwater mechanics. Prentice Hall, Englewood Cliffs

29. Polubarinova-Kochina PY (1962) Theory of ground water movement. Princeton University Press, Princeton

30. Strack ODL (2003) Theory and applications of the analytic element method. Rev Geophys 41(2):1005. doi:10.1029/ 2002RG000111

31. Townley LR (1995) The response of aquifers to periodic forcing. Adv Water Resour 18:125-46

32. Moon P, Spencer DE (1971) Field theory handbook: including coordinate systems, differential equations and their solutions, 2nd edn. Springer-Verlag, New York

33. Bakker M (2006) Where do periodic variations in the discharge of a well become negligible? Ground Water 44(3):478-482

34. Abramowitz M, Stegun IA (1965) Handbook of mathematical functions. Dover, New York

35. Oliphant TE (2006) Guide to NumPy. http://www.trelgol.com

36. Bakker M (2004) Modeling transient flow with wiggly analytic elements. In: Proceedings of computational methods in water resources XV, Chapel Hill, NC, 13-17 June, 2004

37. Temme NM (1996) Special functions. An introduction to the classical functions of mathematical physics. Wiley, New York 